\title{
Radiation-hydrodynamic Simulations of Protostellar Outflows: Synthetic Observations and Data Comparisons
}

\section{Citation}

Offner, Stella S. R., Eve J. Lee, Alyssa A. Goodman, and Héctor Arce. 2011. "RADIATIONHYDRODYNAMIC SIMULATIONS OF PROTOSTELLAR OUTFLOWS: SYNTHETIC OBSERVATIONS AND DATA COMPARISONS." The Astrophysical Journal 743 (1): 91. https:// doi.org/10.1088/0004-637x/743/1/91.

\section{Permanent link}

http://nrs.harvard.edu/urn-3:HUL.InstRepos:41397487

\section{Terms of Use}

This article was downloaded from Harvard University's DASH repository, and is made available under the terms and conditions applicable to Other Posted Material, as set forth at http:// nrs.harvard.edu/urn-3:HUL.InstRepos:dash.current.terms-of-use\#LAA

\section{Share Your Story}

The Harvard community has made this article openly available.

Please share how this access benefits you. Submit a story.

\section{Accessibility}




\title{
RADIATION-HYDRODYNAMIC SIMULATIONS OF PROTOSTELLAR OUTFLOWS: SYNTHETIC OBSERVATIONS AND DATA COMPARISONS
}

\author{
Stella S. R. Offner ${ }^{1}$, Eve J. LeE ${ }^{2}$, Alyssa A. Goodman ${ }^{1}$, and Héctor Arce ${ }^{3}$ \\ ${ }^{1}$ Harvard-Smithsonian Center for Astrophysics, Cambridge, MA 02138, USA; soffner@ cfa.harvard.edu \\ ${ }^{2}$ Department of Astronomy and Astrophysics, University of Toronto, Toronto, ON M5S 3G4, Canada \\ ${ }^{3}$ Department of Astronomy, Yale University, New Haven, CT 06511, USA \\ Received 2011 September 1; accepted 2011 October 25; published 2011 November 23
}

\begin{abstract}
We present results from three-dimensional, self-gravitating, radiation-hydrodynamic simulations of low-mass protostellar outflows. We construct synthetic observations in ${ }^{12} \mathrm{CO}$ in order to compare with observed outflows and evaluate the effects of beam resolution and outflow orientation on inferred outflow properties. To facilitate the comparison, we develop a quantitative prescription for measuring outflow opening angles. Using this prescription, we demonstrate that, in both simulations and synthetic observations, outflow opening angles broaden with time similarly to observed outflows. However, the interaction between the outflowing gas and the turbulent core envelope produces significant asymmetry between the redshifted and blueshifted outflow lobes. We find that applying a velocity cutoff may result in outflow masses that are underestimated by a factor five or more, and masses derived from optically thick CO emission further underpredict the mass of the high-velocity gas by a factor of 5-10. Derived excitation temperatures indicate that outflowing gas is hotter than the ambient gas with temperature rising over time, which is in agreement with the simulation gas temperatures. However, excitation temperatures are otherwise not well correlated with the actual gas temperature.
\end{abstract}

Key words: methods: numerical - molecular data - radiative transfer - stars: formation - stars: winds, outflows

Online-only material: color figures

\section{INTRODUCTION}

Young protostars power high-velocity jets that entrain and unbind a large fraction of the natal protostellar core gas. Outflow mass rates are estimated to be comparable in magnitude to protostellar accretion rates (Bontemps et al. 1996). Consequently, outflows likely play an important role in removing core mass and terminating the accretion process (Matzner \& McKee 2000; Myers 2009). The largest outflows accelerate gas to velocities exceeding $100 \mathrm{~km} \mathrm{~s}^{-1}$ and extend across several parsecs. On these scales, outflows powerfully impact their environment and potentially inject significant energy back into the parent molecular cloud (Matzner \& McKee 1999). This feedback may be partially responsible for maintaining turbulence on 0.1-1 pc scales (Nakamura \& Li 2007; Swift \& Welch 2008; Arce \& Sargent 2005; Arce et al. 2010).

Due to observational resolution limits and the inherently opaque nature of young protostellar cores, the central outflow engine is not well understood. Outflow morphology, velocity distribution, and opening angle provide important constraints for theoretical models. For example, observations suggest that outflows develop from a narrow jet-like morphology into a wide-angle wind (Arce \& Sargent 2006; Seale \& Looney 2008). Outflow velocities also follow a Hubble-like relationship, with the gas velocity increasing linearly from the source (Arce et al. 2007). Among proposed models, the wide-angle-wind model ( $\mathrm{Li}$ $\&$ Shu 1996) and the jet bow-shock model (Chernin \& Masson 1995) appear to best explain the observed outflow characteristics (Lee et al. 2000).

However, a number of uncertainties complicate interpretation of the observations. Orientation of the disk-outflow geometry relative to the line of sight make protostellar age estimations imprecise (Ladd et al. 1998; Robitaille et al. 2006). Once the outflow gas shocks and cools, it quickly mixes with ambient turbulent gas and becomes impossible to distinguish. This means that observed outflow gas may only span the previous few thousand years of the flow. In addition, the ambient lowdensity, high-line-width cloud gas $\left(\simeq 10 \mathrm{~km} \mathrm{~s}^{-1}\right)$ along the line of sight makes identification of a wide-angle, low-velocity outflow component problematic (e.g., Arce et al. 2010).

Precession of the jet due to rotational wobbling of the protostar may increase the broadness and clumpiness of the outflow (Rosen \& Smith 2004). It is likely that variability in the protostellar accretion rate causes variability in outflow properties (e.g., Cernicharo \& Reipurth 1996; Arce \& Goodman 2002). Consequently, bursty accretion may produce a signature in the velocity spectrum and outflow morphology. However, this is difficult to demonstrate observationally.

In this work, we use gravito-radiation-hydrodynamic simulations modeling protostellar outflows to study outflow evolution as a function of time. First, we introduce a quantitative method for determining opening angles and use this to characterize the outflow properties of the raw simulation data. Next, we investigate the dependence on inclination and observed resolution. Finally, we present synthetic observations of the simulations in ${ }^{12} \mathrm{CO}$. Throughout, we compare with interferometric observations of seven protostellar outflows studied by Arce \& Sargent (2006, henceforth AS06). These observations offer a high-resolution picture of the inner outflow regions, the interaction between outflow and envelope, and the evolution of outflow characteristics over time. Since the simulations provide complete three-dimensional information, they allow us to compare position-position-position (ppp) data with synthetic and observational position-position-velocity (ppv) data and examine the observations.

We outline the simulation methodology and initial conditions in Section 2. We define our procedure for characterizing the outflows in Section 3. We present the data analysis, synthetic observations, and observational comparison in Section 4. We summarize our results in Section 5. 


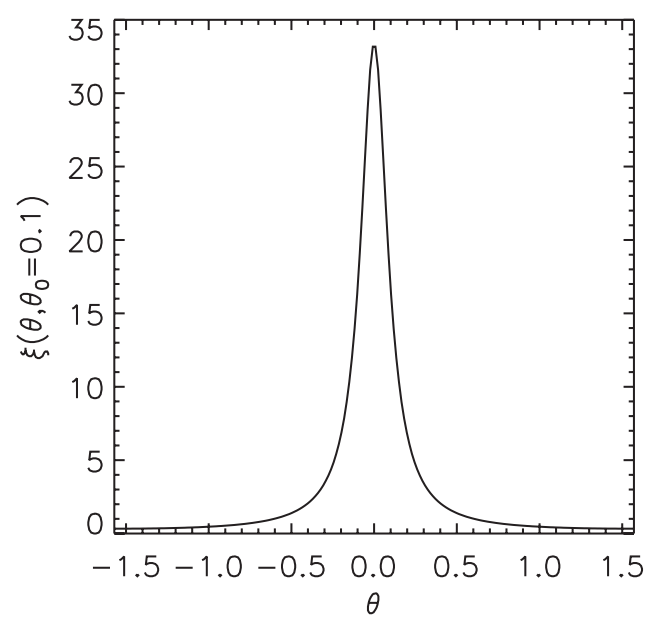

Figure 1. Distribution of injected angular momentum $\xi\left(\theta, \theta_{0}\right)$ vs. polar angle $\theta$, for $\theta_{0}=0.1$

\section{NUMERICAL METHODS}

The simulations are performed using the ORION adaptive mesh refinement (AMR) code. For our study, we use the radiation-hydrodynamic, self-gravitating simulations of Offner et al. (2009b, henceforth OKMK09) as initial conditions.

The OKMK09 simulations have a mean density of $4.46 \times$ $10^{-20} \mathrm{~g} \mathrm{~cm}^{-3}$, three-dimensional Mach number of 6.6, and a total mass of $185 M_{\odot}$. Energy is injected at a constant rate to maintain the level of turbulence (e.g., Stone et al. 1998). Particles are inserted in regions of the flow that exceed the Jeans condition (Krumholz et al. 2004). These stars are endowed with a subgrid stellar evolution model that includes accretion luminosity down to the stellar surface, Kelvin-Helmholz contraction, and nuclear burning. The original calculations include the effects of radiation feedback from the forming stars but neglect protostellar outflows, which is the subject of our investigation. Note that we use the terms outflow and wind interchangeably and do not distinguish between gas that is directly launched near the protostar and gas that is swept up and entrained by this gas.

From the OKMK09 simulations, we select two forming protostars to receive additional refinement beginning prior to their formation. In these high-resolution regions, the minimum cell size is $4 \mathrm{AU}$. The remainder of the simulation is evolved with the previous number of four AMR levels, i.e., 32 AU minimum cell size, and without outflows. Although more computationally expensive than modeling isolated protostars, this method allows us to use self-consistent turbulent initial conditions and follow the evolution of the outflows within a turbulent, clustered star-forming environment. Henceforth, we refer to the two high-resolution runs as $R 1$ and $R 2$.

To the selected protostars, we add a sub-grid model for protostellar winds based upon Matzner \& McKee (1999). Cunningham et al. (2011) describe the details of the model implementation in ORION, which we briefly summarize here. The outflow model is characterized by three dimensionless parameters that specify the outflow ejection efficiency, outflow velocity, and momentum distribution. The mass ejection rate, $f_{w}$, gives the fraction of infalling gas that is accelerated into a wind. This fraction is observationally uncertain, but the disk wind (Pelletier \& Pudritz 1992) and X-wind (Shu et al. 1994) models predict $f_{w} \simeq 0.1-0.33$. Here, we adopt $f_{w}=0.33$. Consequently, $1.0 /\left(1+f_{w}\right)$ of the infalling gas accretes onto the star, while $f_{w} /\left(1+f_{w}\right)$ is launched in an outflow.
The wind launching velocity is given by the Keplerian velocity at the stellar surface, $v_{K}=\sqrt{G M_{*} / r_{*}}$. In the case of high-mass protostars these velocities can exceed $200 \mathrm{~km} \mathrm{~s}^{-1}$, greatly constraining the numerical time step of the calculation. Cunningham et al. (2011) solve this problem by limiting the outflow velocity to a fraction of the Keplerian speed, $f_{v}$. In the calculations we present here, we set $f_{v}=1$ since the stars that form have low mass with Keplerian velocities $\lesssim 100 \mathrm{~km} \mathrm{~s}^{-1}$.

The direction of the outflow ejection is set by the direction of the angular momentum vector of the protostar. This is determined by the angular momentum of the accreting gas, a quantity that depends upon the turbulent properties of the core and evolves over the calculation. Thus, the direction of the wind is not fixed or set as an input parameter but is self-consistently dictated by the hydrodynamic evolution of the accretion flow. As a consequence, the launching axis is roughly parallel to the angular momentum vector of the accretion disk.

We define the effective opening angle of the wind, $\theta_{0}$, following Matzner \& McKee (1999). They characterize the angular distribution of the outflow momentum:

$$
\xi\left(\theta, \theta_{0}\right)=\left[\ln \left(\frac{2}{\theta_{0}}\right)\left(\sin ^{2} \theta+\theta_{0}^{2}\right)\right]^{-1},
$$

where $\theta$ is the polar angle measured from the protostar's rotation axis. Through comparisons with observational data of low-mass protostars Matzner \& McKee (1999) find that $\theta_{0} \lesssim 0.05$ and suggest a fiducial value of $\theta_{0}=0.01$. This value results in a strongly peaked distribution, which numerically deposits nearly all the momentum in a couple cells along the rotational axes. Here, we adopt a slightly larger value of $\theta_{0}=0.1$ as shown in Figure 1, which is comparable to the angular width of an individual fine cell. The momentum assigned to a cell is $\bar{\xi}$, the momentum distribution function averaged over the polar angle subtended by that cell.

The wind is injected into cells with radial distances $4 \Delta x<$ $r \leqslant 8 \Delta x$ from the protostar, so that the wind injection zone lies outside the accretion region. This serves the dual purpose of providing better resolution in the injection region and allowing gas to continue to accrete onto the central protostar. In practice, $\xi$ is also set to 0 when $\theta$ becomes close to $\pi / 2$. Our outflow algorithm is fully mass conserving. Gas that was previously automatically accreted onto the stars in the original simulation is instead divided between accreted gas and outflow gas that is deposited back onto the numerical grid.

Instead of the Pollack et al. (1994) dust opacity model used by OKMK09, we switch to an updated model from Semenov et al. (2003), which assumes a standard iron abundance and treats the grains as composite aggregates. The strong bow shocks produced by outflowing gas running into ambient material can generate temperatures well in excess of the dust destruction temperature. Since the outflows simulated here are young and very low mass, only a small number of cells ever reach temperatures above $1000 \mathrm{~K}$. Nonetheless, we include the treatment for atomic line cooling described by Cunningham et al. (2011), which implicitly solves for cell temperatures exceeding $10^{4} \mathrm{~K}$.

\section{ANALYSIS}

\subsection{Outflow Identification}

Identifying the gas associated with outflowing material remains a key observational challenge. Generally, outflow gas 


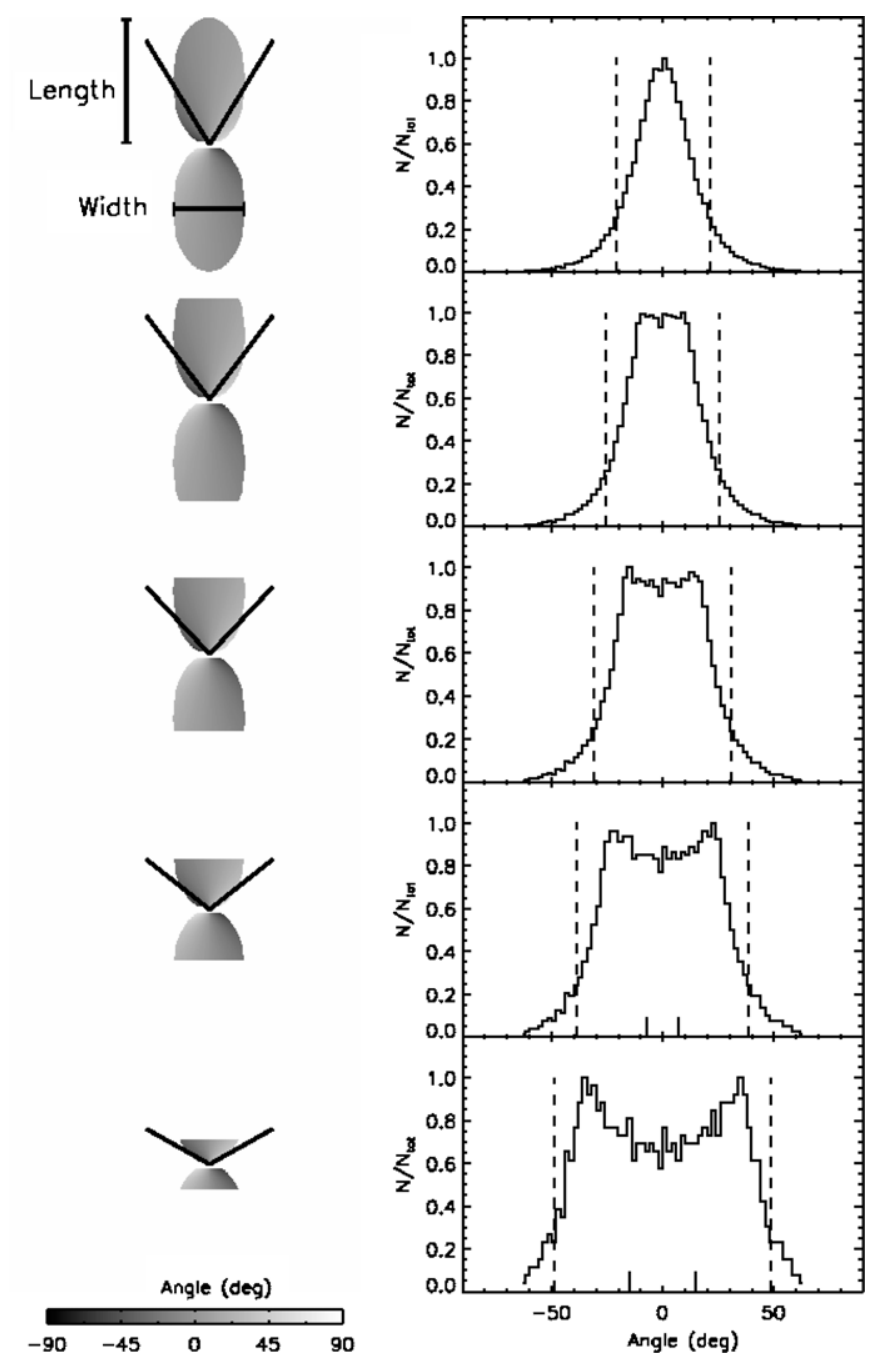

Figure 2. Left: idealized elliptical outflow shape with half-angle $\arctan (b / a)=$ $20^{\circ}$, where $a$ and $b$ are the length and width of the major and minor axes, progressively truncated toward the center. The black lines show the measured opening angle. Right: angle distribution of the pixels constituting the outflow, where the vertical lines indicate the opening angle defined as the full width at quarter-maximum of the distribution.

is higher velocity, hotter, and less dense than the accreting material. It is difficult to use temperature as a means to distinguish outflow gas. In nearby well-resolved regions, it is possible to image outflow cavities in scattered light (Seale \& Looney 2008). However, observers typically use low-density tracers such as ${ }^{12} \mathrm{CO}$ to map the outflow gas, since these data supply both direct velocity information and indirect estimates of the gas mass. Within molecular line datacubes, line-of-sight velocity remains the primary means of identification. In AS06, outflows are defined by selectively integrating over the ${ }^{12} \mathrm{CO}(1-0)$ emission in a range of high-velocity channels determined by visual inspection.

To identify the outflows in the simulations, we set a minimum outflow gas velocity of $2 \mathrm{~km} \mathrm{~s}^{-1}$. We then generate a column density map of cells above this cutoff, which is analogous to an observed intensity map integrated over selected velocity channels.

Figure 2 shows the angle distributions of integrated emission for schematic elliptical outflows. In this two-dimensional space, we treat each outflow lobe independently and characterize outflow morphology using five parameters: opening angle, length, width, shape, and inclination. To measure the opening angle, we calculate the angle with respect to the $z$-axis for each pixel that is above a given velocity minimum. In the analysis, we weight all such pixels equally. The opening angle is then defined to be the full width at quarter-maximum of the angle distribution. This definition is empirically selected to correspond to opening angles derived from intensity maps by eye (and protractor). We derive the outflow inclination with respect to the vertical axis by fitting the angle distribution with either a Gaussian or higher order polynomial. Note that an outflow may also be inclined along the line of sight toward (or away from) the observer. However, our method only fits for the inclination in the plane of the sky. For an elliptical outflow, the inclination is the location of the distribution maximum, while for a more conically shaped outflow, it is the local minimum. Note that since the simulation inclination is obtained from the projected distribution of cells it may not identically correspond to the direction in which the outflow is launched. In addition, interaction between the outflow and the envelope may affect the inferred outflow inclination.

For a given distribution of connected high-velocity cells, the physical length of the outflow is the longest extent measured from the center outward along the outflow major axis. The width is the maximum extent along the axis perpendicular to the outflow length. The Gaussian parameter, Gauss, is the $\chi^{2}$ value of a Gaussian fit to the angle distribution. As illustrated by Figure 2, the distribution of pixels for elliptically shaped outflows is well described by a Gaussian. Our opening angle definition is formulated to be consistent with an elliptical outflow such that $\arctan ($ Width/Length) $\simeq \theta$. As we show in Section 4.2 , the pixels close to the protostar are likely to be dominated by the beam characteristics, and thus are not suitable for an opening angle determination.

\subsection{Opening Angle Measurement}

We first characterize an idealized, symmetric outflow geometry. Figure 2 shows an ellipse of length $0.1 \mathrm{pc}$, which has been superimposed upon the AMR grid cell hierarchy of a simulation output. The effect of truncating the outflow length is apparent in the angle distribution evolution shown on the right. The inferred angle increases with increasing truncation as the wide-angle cells at the base of the ellipse dominate the distribution.

Figure 3 shows the opening angle and major axis inclination for a series of truncated elliptical outflows. The error bars indicate the $\sqrt{N}$ error, where $N$ is the number of beams contained in the ellipse assuming that the outflow is placed $250 \mathrm{pc}$ away and observed with a beam of $4^{\prime \prime}$. Figure 3(a) illustrates that the error bars do not necessarily contain the inferred opening angle for the outflow with full information (i.e., no truncation) even though the uncertainty increases as the outflow length shrinks. This indicates that a poorly resolved outflow may be measured to have a fundamentally different opening angle than the same outflow with complete information. The outflow major axis remains fixed during the truncation. Figure 3(b) demonstrates that the derived direction of the outflow axis is fairly insensitive to the truncation.

For comparison, we re-analyze the AS06 data to obtain the opening angles quantitatively. Figure 4 shows the measured angles for the upper and lower lobes and the mean of the two. The slope of the fit we find to the data, 0.15 , is quite close to the value of $0.16 \pm 0.4$ found by AS06. The intercept of 1.22 is also within error of the previously published value of $1.1 \pm$ 0.2 . This is encouraging since it suggests that our method is a good quantitative alternative to fitting the outflow angles by eye. 


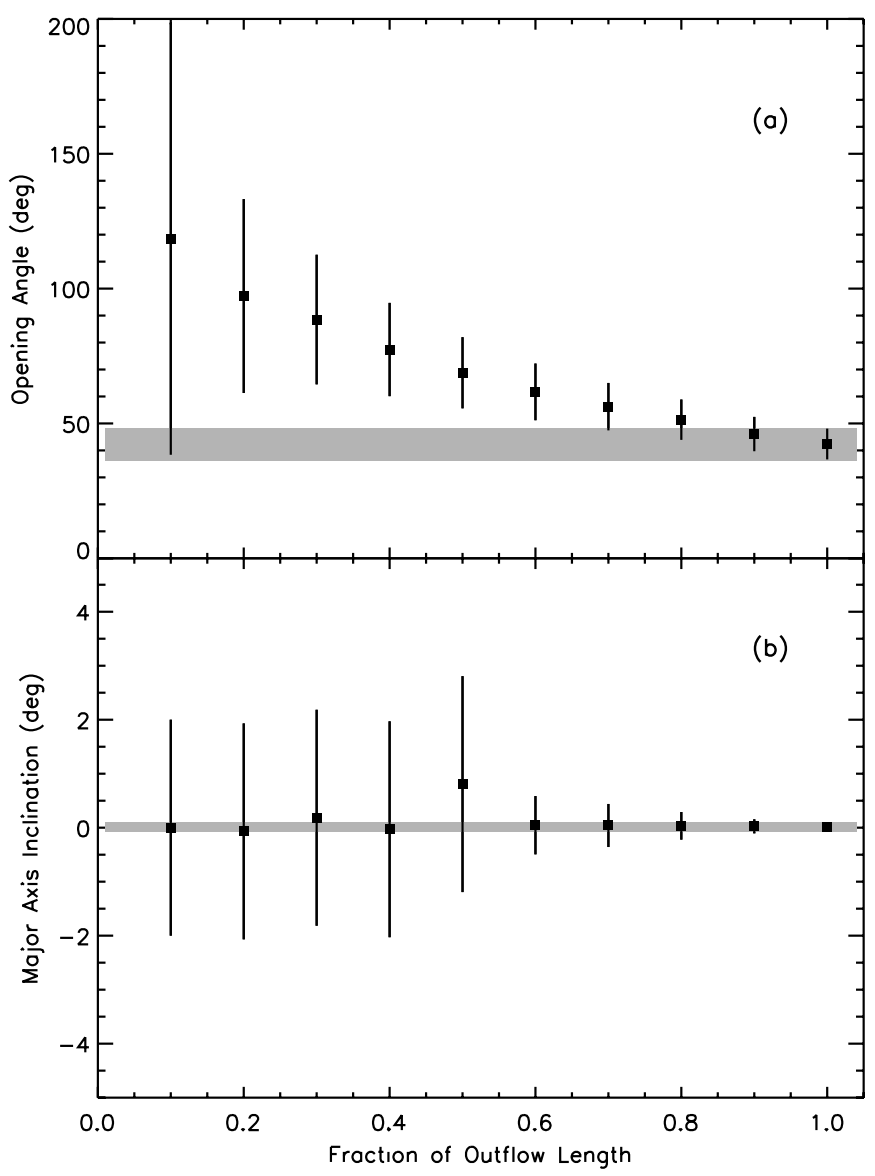

Figure 3. (a) Opening angle as a function of ellipse length included in the angle determination. (Ellipses with fractional lengths $0.2,0.4,0.6,0.8$, and 1.0 are shown in Figure 2.) (b) Inclination of the ellipse major axis relative to the $y$-axis as a function of the ellipse length fraction. The gray shading shows the range for the non-truncated case.

One caveat to the fitting method is that for small numbers of pixels the algorithm becomes sensitive to the bin size. We indicate this uncertainty using vertical error bars on the plot, which are twice the bin size used in the fit.

As shown in the bottom panel of Figure 4, the angle distribution tends to evolve from an elliptical shape (Gauss $\left(\chi^{2}\right) \leqslant 20$ ) to a more conical or irregular shape (Gauss $\left.\left(\chi^{2}\right)>20\right)$. This trend may be partially an artifact of how the outflow is observationally sampled at late times rather than an indication of how the geometry actually changes. This is supported by the apparent decrease of outflow length with time, which is most likely because the older gas has blended with the ambient gas.

\section{RESULTS}

\subsection{Outflow Morphology}

Figure 5 shows a volume rendering of the simulation velocities for both runs. The outflow axis in $R 1$ is almost directly aligned with the $z$-axis so that $v_{z}$ traces the fastest moving gas, which ranges up to $70 \mathrm{~km} \mathrm{~s}^{-1}$. $R 2$ forms two protostars with fairly distinct outflows. The secondary protostar arises from fragmentation in the core rather than disk fragmentation, so that the outflow axes of the two are somewhat misaligned. However, the two $R 2$ protostars are sufficiently close that separating the outflow lobes in projection is difficult. Consequently, in the following analysis we will use $R 1$ to explore outflow evolution for

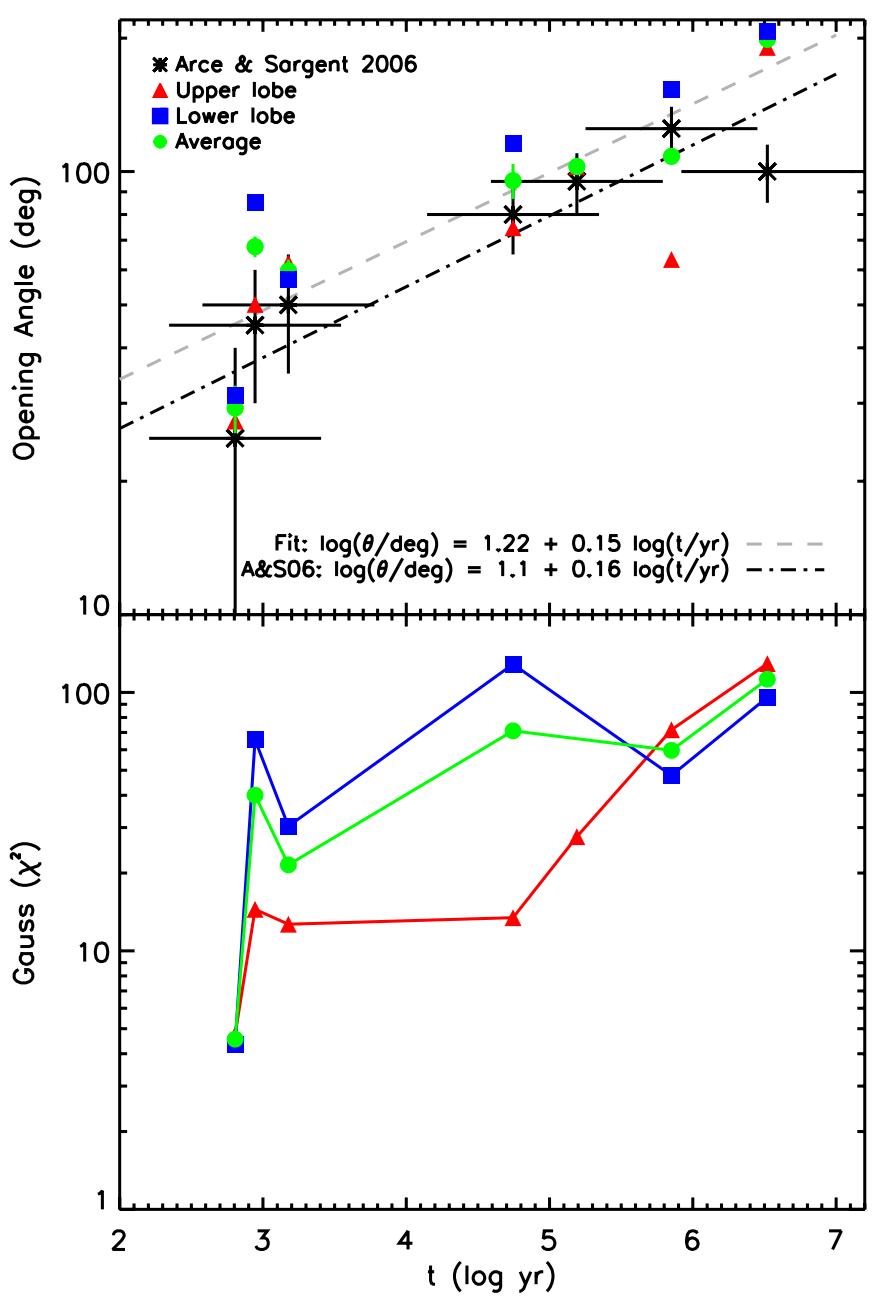

Figure 4. Opening angle (top) and Gauss parameter (bottom) for the Arce \& Sargent (2006) sample as a function of estimated age. Stars indicate the original values derived by Arce \& Sargent (2006) with the fit shown by the dot-dashed line. The vertical error bars on the average opening angle are twice the angle bin size used for the fit; in some cases they are smaller than the symbol. The dashed line shows the revised fit of the mean opening angle derived for our angle definition.

(A color version of this figure is available in the online journal.)

individual sources, while $R 2$ will illustrate a case in which observations are confused by the presence of a second, unidentified outflow source.

All three outflows shown in Figure 5 exhibit a significant amount of asymmetry between the lobes. The sub-grid model launches the outflow gas from the grid symmetrically about the protostars. (On the scale of the figure the outflow launching region is contained in the central pixel.) This means that any asymmetry that arises is directly the result of the interaction between the outflow and the turbulent envelope. For example, the $R 1$ protostar forms in a more filamentary structure and the net angular momentum direction is almost directly aligned with the filament axes. As a result, the positive $z$ outflow lobe is diverted from the launching axis and confined by the dense filament gas. The outflow lobe along the negative $z$-axis breaks out of the filament and extends further. In $R 2$ asymmetry in the core also leads to mismatched outflow lobe sizes.

\subsection{Opening Angle Evolution}

Figure 6 shows the $R 1$ outflow evolution as a function of time. The outflow gas is identified using a velocity cutoff of 

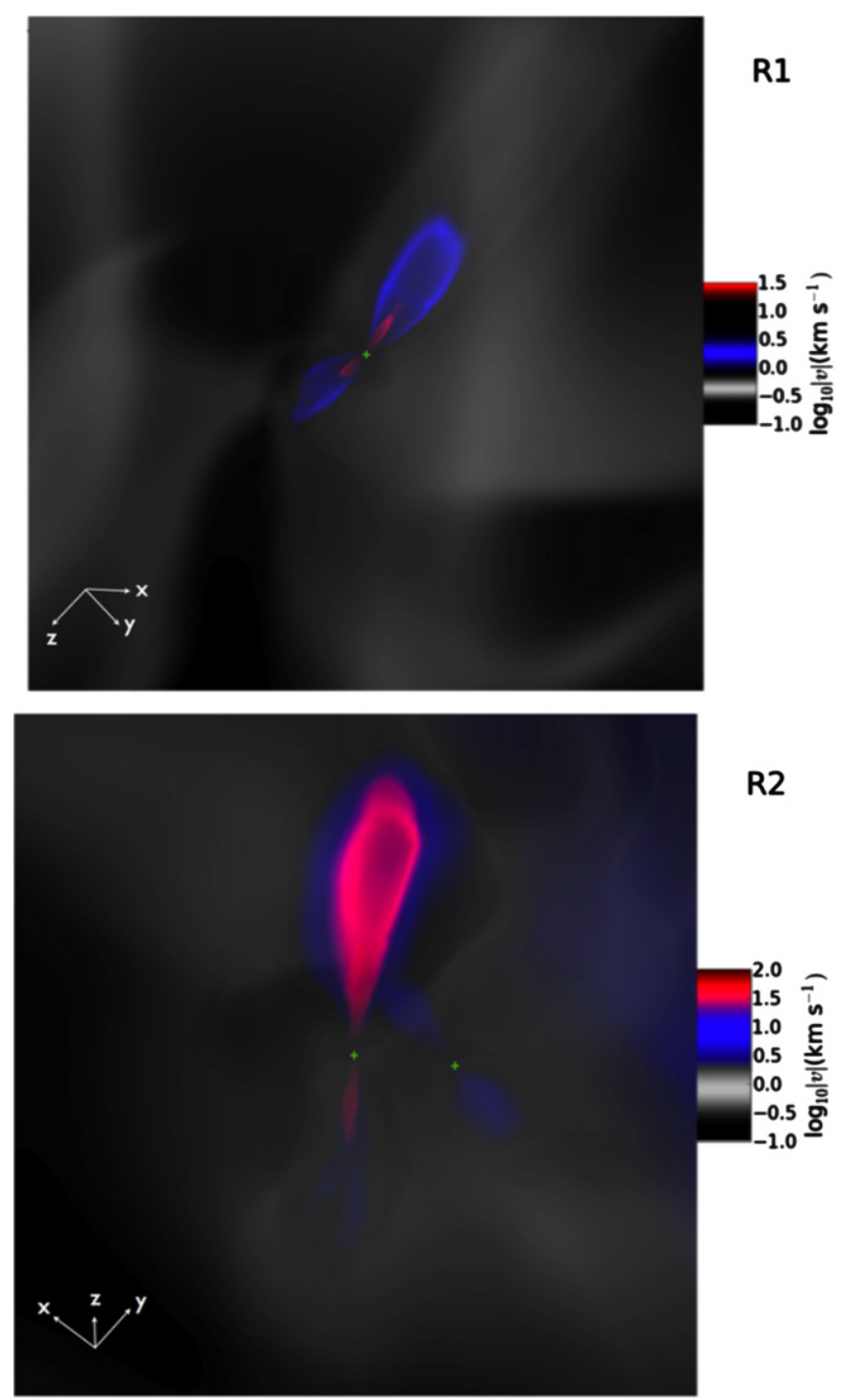

Figure 5. Volume rendering of the outflow gas velocity $\left(\mathrm{km} \mathrm{s}^{-1}\right)$ for $R 1$ at $40 \mathrm{kyr}$ (top) and for $R 2$ at $20 \mathrm{kyr}$ (bottom). The box is $0.15 \mathrm{pc}$ on a side with the stellar positions marked by green crosses.

(A color version of this figure is available in the online journal.)

$2 \mathrm{~km} \mathrm{~s}^{-1}$, where the full three-dimensional information is used to determine the outflow and envelope masses. These cells are projected along the $x$-direction so that the outflow opening angle, width, and length are calculated when the outflow axis is nearly parallel to the $z$-axis. A fit to the opening angles demonstrates that the lobes widen with time similarly to observations. The earliest time only contains a small number of cells in the outflow so that it is artificially broadened, but a strong trend is apparent for $3.7 \leqslant \log (t) \leqslant 4.7$. (See Section 5 for discussion of the origin of this broadening.) However, the different outflow lobes in Figure 6 differ from one another and display significant shorter-time variation. For example, the upper outflow lobe growth stalls at late times. This suggests that even if outflows generally evolve with time, variation in individual outflow behavior can be large.

Considering the large uncertainties in observed outflow ages, poorly constrained line-of-sight inclinations, and instrument limitations, the agreement between the simulations and the observations shown in Figure 4 is striking. This supports the finding of AS06 that outflow angles evolve with time. However, the protostellar masses in these calculations are $\lesssim 0.1 M_{\odot}$ for the time of comparison, so they are still very young. Outflow sizes are $\lesssim 0.1 \mathrm{pc}$, although this is similar to the sizes of those in AS06, which are $\sim 0.005-0.1 \mathrm{pc}$. Figure 6 illustrates the length of only the connected cells with velocities $\geqslant 2 \mathrm{~km} \mathrm{~s}^{-1}$. These cells are also very low density and have a total mass of $\sim 0.001 M_{\odot}$.

The $R 1$ opening angle extrapolated at $t=0$ is lower than that found by AS06, although the positive $z$ fit is still within the observational error. Smearing due to the observational beam likely accounts for some of this discrepancy. Figure 7 shows the measured opening angles for $R 1$ as a function of convolution with different beam sizes. For a $4^{\prime \prime}$ beam, which is comparable to the resolution of AS06, some angles may be broadened by up to $\sim 80 \%$. Broadening becomes more significant for larger beam sizes. The earliest times in the simulation, for which the outflow is already broadened by the small number of cells, appear least affected by the beam smearing.

\subsection{Synthetic Observations}

While comparison between the simulation and observed ${ }^{12} \mathrm{CO}$ data in Section 4.2 is suggestive, it is important to compare the data directly with synthetic observations in ${ }^{12} \mathrm{CO}$. In this section we use the radiative transfer code MOLLIE (Keto \& Rybicki 2010) to model the emission from the first seven ${ }^{12} \mathrm{CO}$ and ${ }^{13} \mathrm{CO}$ transitions. We assume a standard abundance of $5.6 \times 10^{-5}{ }^{12} \mathrm{CO}$ relative to $\mathrm{H}_{2}$ and an abundance of $7.3 \times 10^{-5}{ }^{13} \mathrm{CO}$ relative to $\mathrm{H}_{2}$, which are comparable to $\mathrm{CO}$ estimated abundances in low-mass envelopes and outflows (Carolan et al. 2008, 2009). However, there are a wide range of inferred $\mathrm{CO}$ abundances among different cores and star-forming regions, values of which are often individually uncertain by a factor of two or more. These uncertainties complicate the estimation of gas mass beyond the already challenging problem of identifying the outflow gas and accounting for projection.

We perform the radiative transfer calculation on a cube of side $0.15 \mathrm{pc}$ centered on the protostar. We regrid the AMR data to produce a grid of $128^{3}$ with 130 AU cell resolution nested within a grid of $128^{3}$ and $260 \mathrm{AU}$ cell resolution.

Figure 8 shows integrated ${ }^{12} \mathrm{CO}(1-0)$ emission maps from observations of $R 1$ at different inclinations with respect to the line of sight. This includes all gas with velocities in the range $\pm 20 \mathrm{~km} \mathrm{~s}^{-1}$, which encompasses the minimum and maximum outflow velocity at 130 AU resolution. ${ }^{4}$ The outflow structure is clearly visible in the integrated maps. Such structure may not be apparent in observed integrated maps due to emission from ambient cloud gas between the source and the observer. The right panels show the map an observer would make when integrating over gas in higher velocity channels $\left(|v| \geqslant 2 \mathrm{~km} \mathrm{~s}^{-1}\right)$.

The lower outflow lobe shows substructure that could be interpreted as the result of accretion bursts. However, the accretion history, and hence the outflow mass ejection rate, is fairly smooth (see Figure 12). Instead, the discontinuous morphology results from the outflow interacting with the turbulent and asymmetric core gas.

Figure 9 shows the opening angle versus inclination measured from the ${ }^{12} \mathrm{CO}$ emission maps with no beam smearing. The opening angle appears slightly broader as the outflow axis becomes more parallel to the line of sight, but there is not a strong dependence on the inclination. At low inclinations,

\footnotetext{
4 In the central launching region in which the AMR resolution is $4 \mathrm{AU}$, velocities reach $\sim 100 \mathrm{~km} \mathrm{~s}^{-1}$. These velocities occur in a small volume and are reduced by the flattening to constant pixel size.
} 

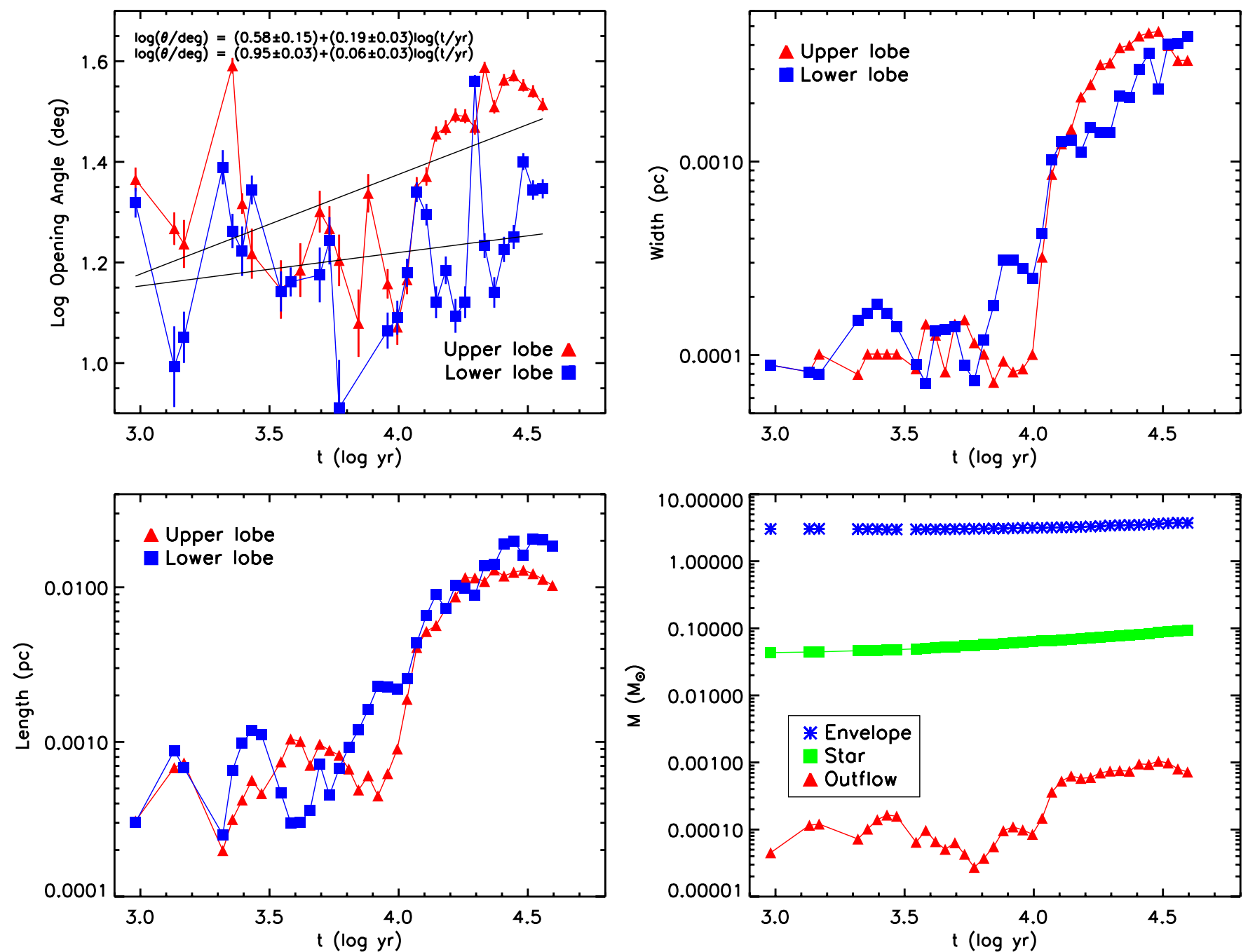

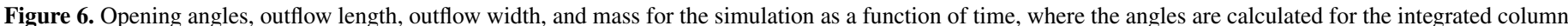

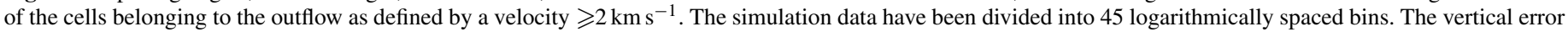

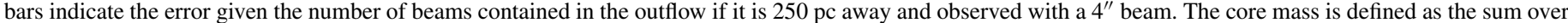
cells with densities $>10^{4} \mathrm{~g} \mathrm{~cm}^{-3}$.

(A color version of this figure is available in the online journal.)

the highest velocities are mainly perpendicular to the line of sight and the angle is measured using only a small sample of cells. When the outflow motion is mostly along the line of sight, the lobes become shorter and rounder. Observationally, outflow orientations with completely overlapping lobes are not well suited to measuring opening angles compared to those with intermediate inclinations. Although the outflow inclination is difficult to infer from projection, the axes of the outflows investigated by AS06 are likely inclined between $30^{\circ}$ and $60^{\circ}$ with respect to the line of sight.

Figure 10 shows the opening angle versus time using the $\mathrm{CO}$ intensity maps to calculate the angle. Like Figure 6, the angle increases on average as a function of time. Linear fits to the average opening angles in Figure 10 give $\theta=0.80+0.18 \log (t)$ and $\theta=0.53+0.25 \log (t)$ for inclinations of $30^{\circ}$ and $45^{\circ}$, respectively. However, like Figure 6 , the two lobes show different trends with time. For the projected data, the lower outflow lobe is nearly flat as a function of time, while the upper lobe widens significantly. This is mainly due to the concurrent widening and elongation of the lower lobe. Despite the variation, this suggests that trends inferred from molecular ppv information are reflective of the actual ppp information. In this case, the similarity is particularly strong because $\mathrm{CO}$ is an efficient tracer of the low-density gas within the outflow cavity.

\subsection{Outflow Mass Evolution}

Molecular hydrogen has no dipole moment and is observationally invisible unless the gas is strongly shocked (Yu et al. 1999). Consequently, CO, which is the next most abundant molecular species, serves as the primary tracer of molecular gas. In this section we consider two methods for estimating molecular gas mass from $\mathrm{CO}$ emission.

For an assumed abundance of $\mathrm{CO}$, a conversion factor can be used to relate $\mathrm{CO}$ intensity to total gas mass. This quantity, known as the $X$-factor, is defined as

$$
X_{\mathrm{CO}}=\frac{N\left(\mathrm{H}_{2}\right)}{W\left({ }^{12} \mathrm{CO}\right)},
$$

where $W\left({ }^{12} \mathrm{CO}\right)$ is the ${ }^{12} \mathrm{CO}(1-0)$ integrated intensity and $N\left(\mathrm{H}_{2}\right)$ is the column density of molecular hydrogen. The abundance of $\mathrm{CO}$ depends upon the local gas density, temperature, and UV field, so that in reality, the $X$-factor varies widely over an individual cloud (Pineda et al. 2008; Glover \& Mac Low 


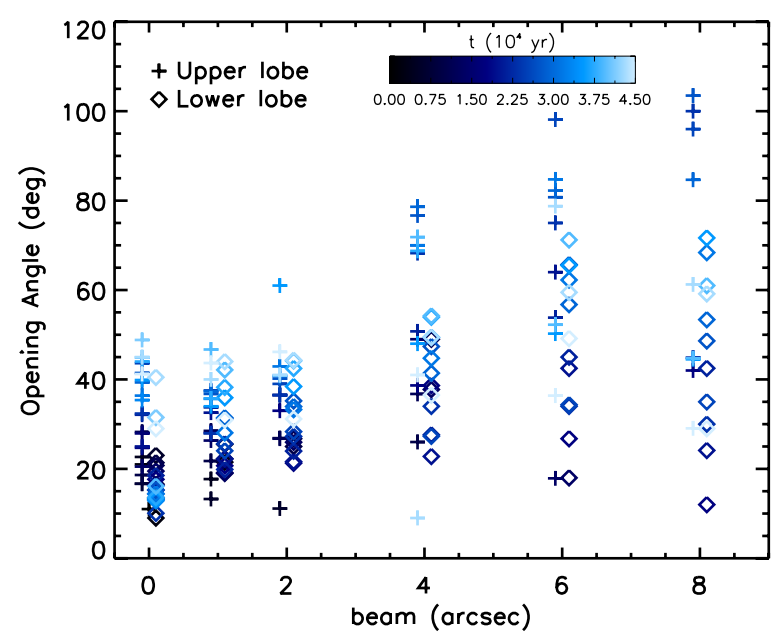

Figure 7. Opening angle vs. beam size assuming that the outflow is observed at a distance of $250 \mathrm{pc}$. The color scale indicates the age of the outflow in units of $10^{4}$ years. Symbols for the different lobes have been offset for clarity.

(A color version of this figure is available in the online journal.)

2011) However, this complicated chemistry is usually reduced to an average $X$-factor. As discussed in Section 4.3, we adopt a typical mean $\mathrm{CO}$ abundance from observations. Here, we use the simulated outflows to assess how accurately the standard $X$-factor and other more complicated methods recover outflow mass.

We adopt $X_{\mathrm{CO}}=1.8 \times 10^{20} \mathrm{~cm}^{-2} \mathrm{~K}^{-1} \mathrm{~km} \mathrm{~s}^{-1}$, which is the mean value for molecular clouds in the solar neighborhood (Dame et al. 2001). Pineda et al. (2008) found that this factor overestimated the gas mass by a factor of $45 \%$ compared to the estimated extinction mass. However, the typical mean abundances inferred by Pineda et al. (2008) are a factor of $\sim 10-30$ higher than the value we assume for our molecular line transfer calculation, where the difference is due to lower expected abundances for dense core and outflow gas versus lower density cloud gas (e.g., Carolan et al. 2008).

Pineda et al. (2008) found that the $X$-factor is most reliable when the gas is both optically thin and $A_{v}>4$. While the emission from the outflow gas alone is marginally optically thin (with increasing optical depth as the inclination approaches $90^{\circ}$ and lower velocities), the integrated ${ }^{12} \mathrm{CO}(1-0)$ emission through the core envelope is optically thick with $\tau>10$. This means that the emission is saturated and $X_{\mathrm{CO}}$, which assumes a linear relationship between emission and gas mass, will underestimate the outflow gas mass.

Bally et al. (1999) and Yu et al. (1999) present a more nuanced approach for obtaining the outflow gas mass from $\mathrm{CO}$ emission. Their methods are similar to that of Arce \& Goodman (2001, henceforth AG01) which we follow here. All three techniques improve upon the estimation above by correcting for optical depth effects and including more of the low-velocity outflow gas mass. We outline the AG01 procedure below and post-process the simulations accordingly (see AG01 and references therein).

While the ${ }^{12} \mathrm{CO}(1-0)$ emission may be quite optically thick, it is possible to utilize the more optically thin ${ }^{13} \mathrm{CO}(1-0)$ emission to calculate the true optical depth and correct the ${ }^{12} \mathrm{CO}(1-0)$ mass estimate. In the first step of the procedure, we spatially average over all the ${ }^{12} \mathrm{CO}(1-0)$ and ${ }^{13} \mathrm{CO}(1-0)$ spectra and derive the ratio of the two lines, $R_{12 / 13}$, as a function of velocity. The resultant parabolic line ratio shape indicates that the opacity is not constant with velocity as many studies assume.

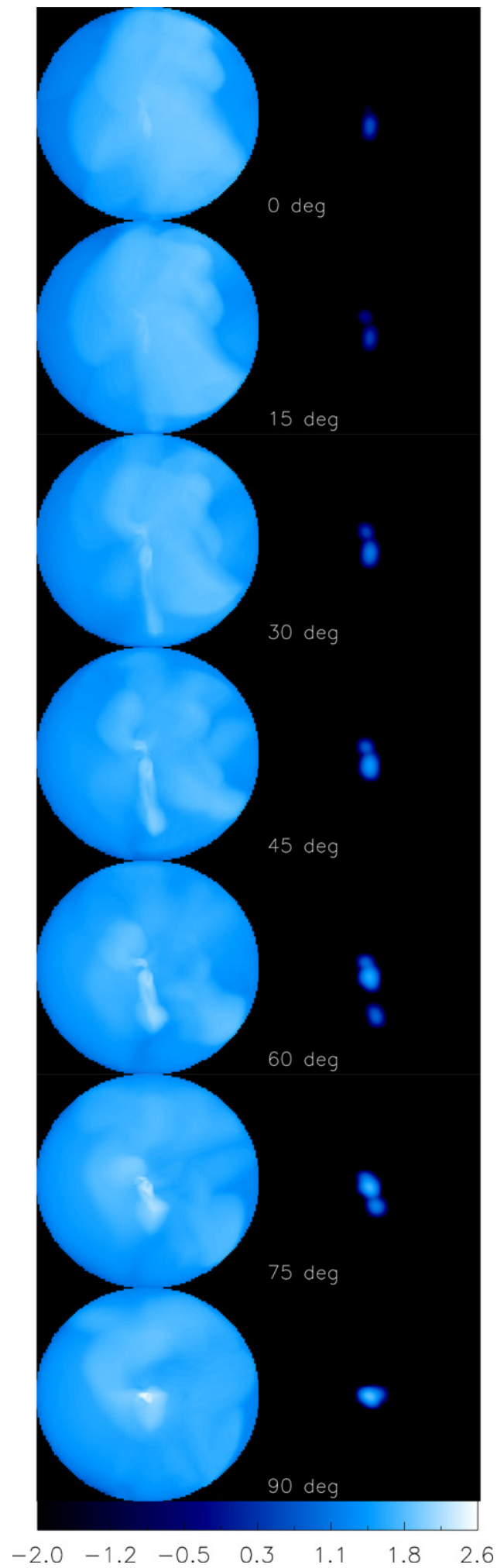

Figure 8. $\log \mathrm{CO}$ intensity $(\mathrm{K})$ for $R 1$ at $t=48 \mathrm{kyr}$. Inclination with respect to the line of sight decreases vertically. The diameter of each map is $0.15 \mathrm{pc}$. The left column shows maps including all gas, while the right column shows integrated mass only for the outflow gas $\left(\left|v-v_{*}\right| \geqslant 2 \mathrm{~km} \mathrm{~s}^{-1}\right)$. On the right, the data have been convolved with a $4^{\prime \prime}$ beam assuming that the outflow is located at $250 \mathrm{pc}$.

(A color version of this figure is available in the online journal.)

We then perform a polynomial fit of $R_{12 / 13}(v)$, which is constrained to reach a minimum at the cloud velocity. Following AG01, who limit the velocity range to exclude a secondary 


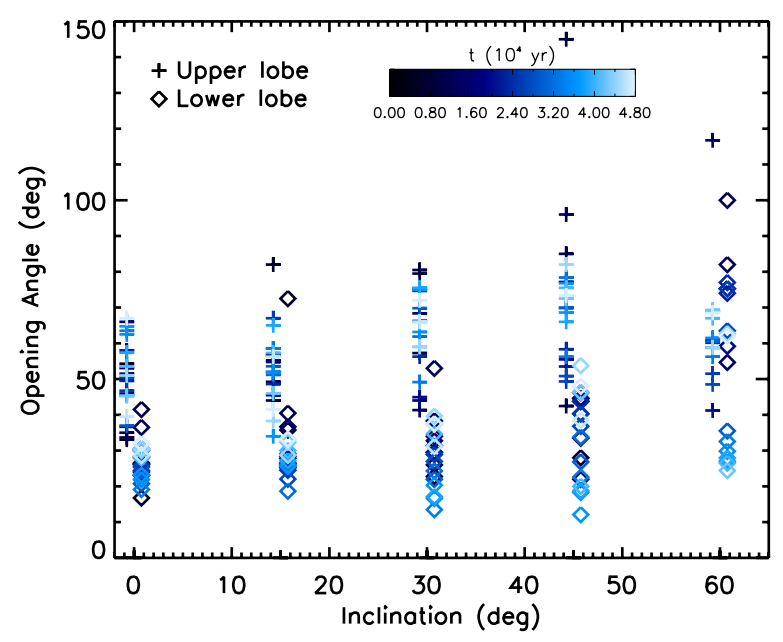

Figure 9. Opening angle vs. inclination (see Figure 7) assuming that the outflow is observed in ${ }^{12} \mathrm{CO}$ at a distance of $250 \mathrm{pc}$. The color scale indicates the age of the outflow in units of $10^{4}$ years. Symbols for the different lobes have been offset for clarity.

(A color version of this figure is available in the online journal.)

coincident cloud, we limit the fitted velocity range to within $1.5 \mathrm{~km} \mathrm{~s}^{-1}$ of the minimum. Using this fit, we extrapolate to the high-velocity wings, where observationally ${ }^{13} \mathrm{CO}$ is too weak to be detected. (Even in the noiseless synthetic observations, the ${ }^{13} \mathrm{CO}$ emission is negligible at velocities above a few $\mathrm{km} \mathrm{s}^{-1}$.)

We next derive an effective "13 $\mathrm{CO}$ main beam temperature," $T_{\mathrm{mb}}^{13}(x, y, v)$, to estimate the ${ }^{13} \mathrm{CO}$ opacity. Observationally, if the ${ }^{13} \mathrm{CO}(1-0)$ emission is greater than twice the rms noise, then $T_{\mathrm{mb}}^{13}(x, y, v)$ can be used directly. Otherwise, the ${ }^{13} \mathrm{CO}$ main beam temperature must be inferred from ${ }^{12} \mathrm{CO}: T_{\mathrm{mb}}^{13}(x, y, v)=$ $T_{\mathrm{mb}}^{12}(x, y, v) / R_{12 / 13}\left(v_{i}\right)$. We adopt an rms noise value of $0.06 \mathrm{~K}$ per $0.2 \mathrm{~km} \mathrm{~s}^{-1}$ channel, similar to that used by AG01.

If ${ }^{13} \mathrm{CO}(1-0)$ is indeed optically thin, then its opacity may be estimated as follows:

$$
\tau_{13}(x, y, v)=-\ln \left(1-\frac{T_{\mathrm{mb}}^{13}(x, y, v)}{T_{0} /\left[\exp \left(T_{0} / T_{\mathrm{ex}}\right)-1\right]-0.87}\right),
$$

where $T_{0}=h v / k=5.29$ and $T_{\mathrm{ex}}$ is the excitation temperature under the assumption that ${ }^{12} \mathrm{CO}(1-0)$ is optically thick:

$$
T_{\mathrm{ex}}=\frac{5.53}{\ln \left[1+5.53 /\left(T_{\text {peak }}+0.82\right)\right]} \text {. }
$$

Here, $T_{\text {peak }}$ is the peak temperature for the ${ }^{12} \mathrm{CO}$ emission. We find $T_{\mathrm{ex}} \sim 10-12 \mathrm{~K}$, in good agreement with the known simulation ambient gas temperature of $10 \mathrm{~K}$.

Finally, we derive the column density of ${ }^{13} \mathrm{CO}$ for each pixel:

$$
N_{13}(x, y, v)=2.42 \times 10^{14}\left(T_{\mathrm{ex}}+0.88\right) \frac{\tau_{13}(x, y, v) d v}{1-\exp \left(-T_{0} / T_{\mathrm{ex}}\right)},
$$

where $d v$ is the channel width in $\mathrm{km} \mathrm{s}^{-1}$. The total mass per pixel of area $A$ is given by

$$
M(x, y, v)=m_{\mathrm{H}_{2}} N_{\mathrm{H}_{2}}(x, y, v) A,
$$

5 Alternatively, for optically thin gas the column density can be expressed as

$$
N_{13}(x, y, v)=\frac{2.42 \times 10^{14}\left(T_{\mathrm{ex}}+0.88\right) T_{\mathrm{mb}}^{13}(x, y, v) d v}{\left[1-\exp \left(-T_{0} / T_{\mathrm{ex}}\right)\right] \times\left[J\left(T_{\mathrm{ex}}\right)-J\left(T_{\mathrm{bg}}\right)\right]},
$$

where $J(T)=T_{0} /\left[\exp \left(T_{0} / T\right)-1\right]$ and $T_{\mathrm{bg}}=2.73 \mathrm{~K}$ is the background temperature. We find that this lowers the derived mass estimate by $\sim 10 \%$.

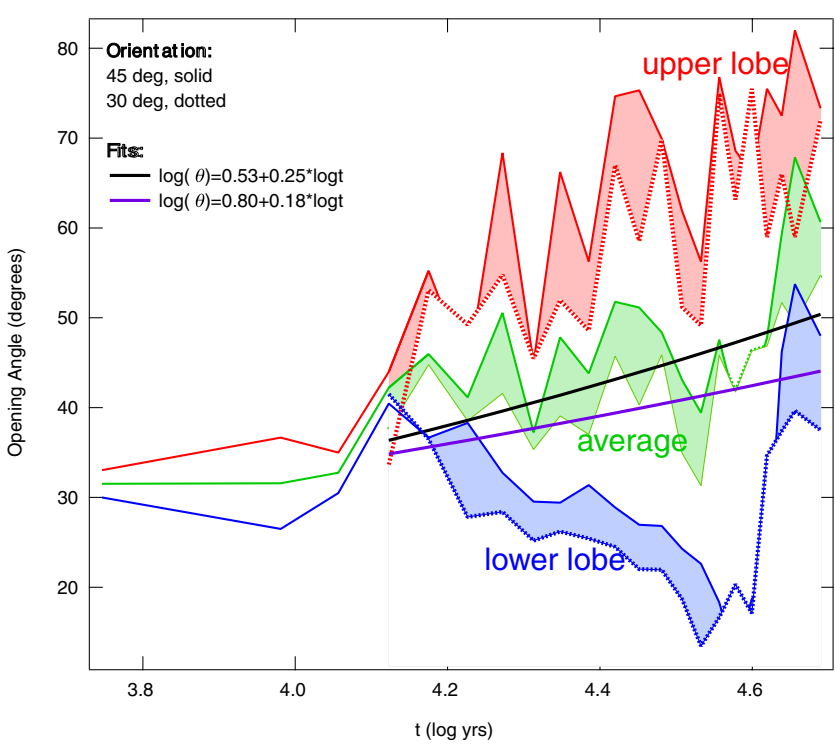

Figure 10. $R 1$ opening angles as a function of time, where the angle is calculated using the integrated ${ }^{12} \mathrm{CO}(1-0)$ intensity map including emission from channels with $|v| \geqslant 2 \mathrm{~km} \mathrm{~s}^{-1}$ and the beam size is $1^{\prime \prime}$. The thick and thin lines indicate $45^{\circ}$ and $30^{\circ}$ inclinations, respectively, relative to the line of sight.

(A color version of this figure is available in the online journal.)

where $m_{\mathrm{H}_{2}}=2.72 m_{\mathrm{H}}$ is the mean molecular weight and the conversion between the ${ }^{13} \mathrm{CO}$ column density and the molecular hydrogen column density is assumed to be $N_{\mathrm{H}_{2}}=1.4 \times 10^{6} N_{13}$. Note that the exact value depends upon the local $X$-factor. When modeling the HH300 outflow in the Taurus star-forming region, AG01 adopt $N_{\mathrm{H}_{2}}=7 \times 10^{5} N_{13}$, a generic value for dense gas in Taurus that was obtained by Frerking et al. (1982).

An additional step is necessary to distinguish between cloud mass and outflow mass. Summing over the total area, we obtain $M(v)$ as shown in Figure 11. AG01 found that the peak, which includes the bulk of the core mass, was well fitted by a Gaussian. We find that a Gaussian provides a satisfactory fit in the case of $R 1$, where there is only a single outflow, while the $R 2$ data, in which there is a second "hidden" outflow, a Gaussian does not well describe all of the high-density gas (see Figure 11). We then subtract this fit from $M(v)$ and integrate over all velocities to get the outflow mass, i.e., the shaded area shown in Figure 11.

Figure 12 shows the simulated outflow mass compared with that derived using the $X$-factor and the AG01 method. For $R 2$, Figure 12 displays the total mass ejected by both stars. Although the axes of the two $R 2$ outflows are not aligned, projection, beam resolution, close proximity, and the low velocities of the outflows would prohibit individual identification of the two outflows observationally.

In the figure, the "total ejected mass" is the total mass launched by the protostar according to the outflow model. This is an essentially numerical quantity representing the total mass deposited in the zones around the protostar as described in Section 2. This quantity is distinct from what an observer would identify as the "outflow mass," which is defined on the basis of velocity and thus also includes entrained gas. At any given time, the launched mass includes gas that goes on to comprise a lowvelocity, less collimated component of the outflow and gas that mixes with the ambient gas becoming indistinguishable even with full information. ${ }^{6}$ Since the launched material is placed on

\footnotetext{
6 Some code methodologies, such as smooth particle hydrodynamics, permit the user to track individual fluid elements, but ORION does not have this capability.
} 

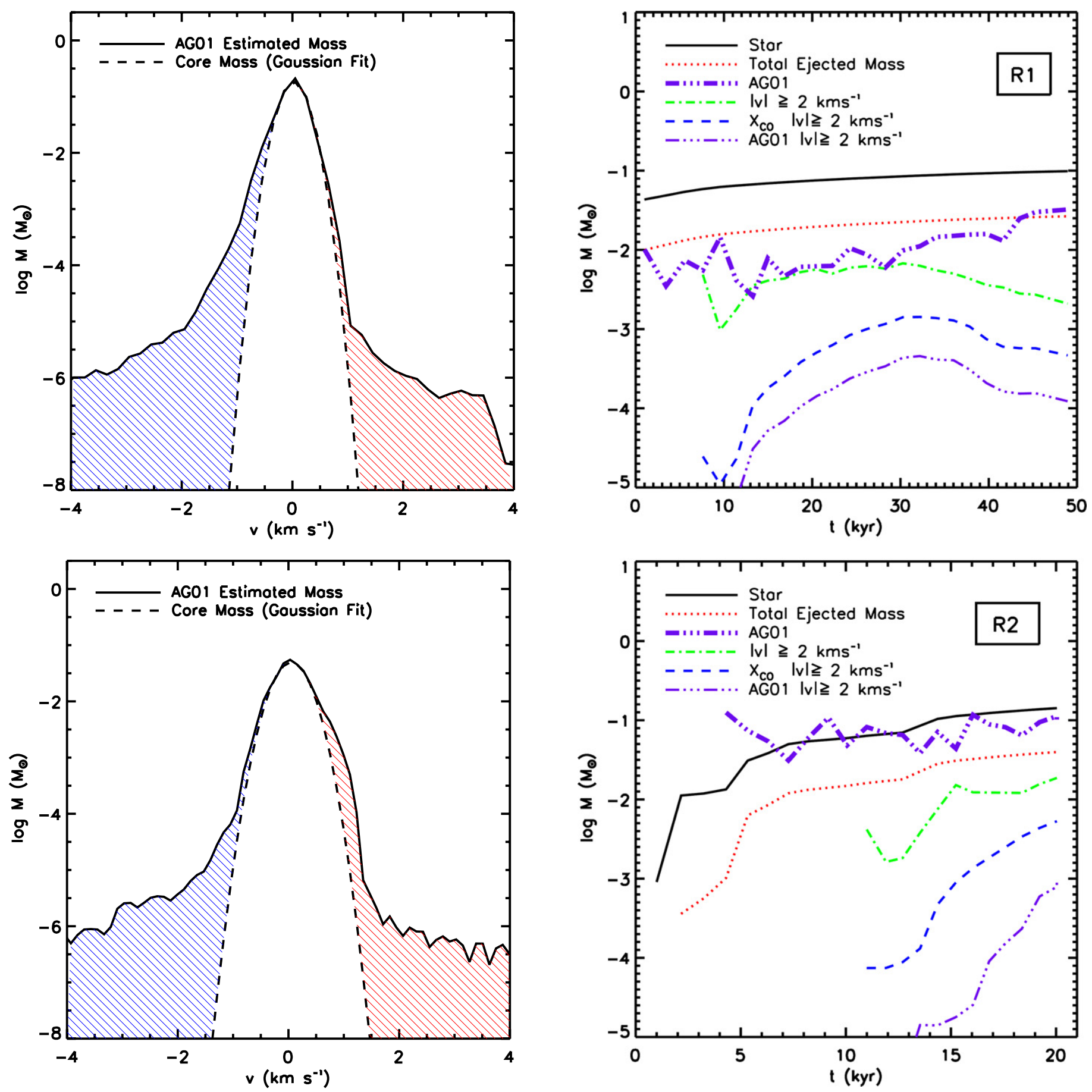

Figure 11. $R 1$ (top) and $R 2$ (bottom) gas mass vs. velocity derived using the AG01 method. A Gaussian fit over the velocity range $-0.75 \mathrm{~km} \mathrm{~s}^{-1} \leqslant$ $v \leqslant 0.75 \mathrm{~km} \mathrm{~s}^{-1}$ (dashed line) represents the non-outflow material. The shaded region shows the blueshifted and redshifted outflow mass. See AG01 (Figure 7(c)) for comparison.

(A color version of this figure is available in the online journal.)

the grid near the protostar by design, this total does not include entrained envelope gas. Once the wind is deposited in the grid it is impossible to differentiate launched gas from entrained, accelerated gas. In principle, due to the addition of entrained gas, the total mass of high-velocity gas, i.e., the outflow mass, could exceed the mass numerically ejected by the protostars.

For both runs, the mass derived from the raw simulation data with $|v| \geqslant 2 \mathrm{~km} \mathrm{~s}^{-1}$ and outflow orientation of $45^{\circ}$ is

Figure 12. Star and outflow gas mass as a function of time for the outflows inclined approximately $45^{\circ}$ with respect to the line of sight. The total ejected mass (red dotted line) includes all mass ejected over all velocities in the outflow. The outflow mass (green dot-dashed line) is estimated from the raw simulation data for $|v| \geqslant 2 \mathrm{~km} \mathrm{~s}^{-1}$. The $X_{\mathrm{CO}}$ mass (blue dashed line) is estimated from the ${ }^{12} \mathrm{CO}(1-0)$ emission observed with a $4^{\prime \prime}$ resolution beam in channels with $|v| \geqslant 2 \mathrm{~km} \mathrm{~s}^{-1}$. The thick purple dot-dot-dot-dashed line shows the mass estimated via the AG01 method where a fit to the core gas mass is subtracted (see Figure 11). The mass estimated from the AG01 method where a simple velocity cut of $2 \mathrm{~km} \mathrm{~s}^{-1}$ is imposed in lieu of a fit is also shown (thin purple dot-dot-dot-dashed line). Note that the $R 2$ mass estimates include the totals for both stars.

(A color version of this figure is available in the online journal.)

significantly less than the total ejected mass by a factor of 5-10. This reinforces the point that the majority of outflow gas in an 
inclined outflow is simply not observable even with a perfect method for converting between $\mathrm{CO}$ emission and total gas mass. However, the integrated ejected outflow mass does not include gas that is entrained and accelerated by the outflowing material, so in principle the simulated and observed gas could be larger than the total ejected mass.

The gas mass estimated from the $\mathrm{CO}$ emission using the $X$-factor appears to track the mass of the high-velocity gas fairly well, although it is generally a factor of 5-10 lower. This discrepancy is due to the high mean optical depth of the core ( $\tau>10$ for ${ }^{12} \mathrm{CO}(1-0)$ and $\tau \sim$ a few for $\left.{ }^{13} \mathrm{CO}(1-0)\right)$. The AG01 method, which subtracts off the core mass, appears to perform somewhat better for most simulation outputs. However, the accuracy of the mass estimation strongly depends upon how well $M(v)$ can be fitted by a simple function. For example, at late times the $R 1 M(v)$ is slightly asymmetric, leading to a negative derived outflow mass. Note that Figure 11 shows the $\log$ of the mass, whereas the fit is dominated by a few points around $v \simeq 0 \mathrm{~km} \mathrm{~s}^{-1}$. Differences between the gas mass and fit at these low speeds far exceed the total mass contributed by high-velocity gas in the distribution wings. To account for this, we only subtract the curves beyond the full width at halfmaximum of the Gaussian fit and omit velocities where the difference is negative. However, since the mass in the core is so much higher than the mass in the high-velocity channels, the mass estimates strongly reflect the symmetry and Gaussianity of the mass distribution. The success of AG01 in estimating the mass of outflow HH300 relies upon the goodness of fit of a Gaussian and the relatively larger outflow mass relative to the core mass.

If the outflow mass is instead defined as a sum over $M(v)$ where $|v| \geqslant 2 \mathrm{~km} \mathrm{~s}^{-1}$ then the mass estimate follows a similar trend to the mass derived using the $X$-factor. However, this suggests that the more complicated AG01 procedure still underestimates the total outflow mass in the optically thick case. The factor of $\sim 5$ discrepancy between the two methods is due to different assumptions about $\mathrm{CO}$ abundance.

\subsection{Outflow Temperature}

Observationally, ${ }^{12} \mathrm{CO}(1-0)$ and ${ }^{12} \mathrm{CO}(2-1)$ may be used in combination to derive the excitation temperature, $T_{\mathrm{ex}}$. Temperature variation may be used to discriminate between different outflow models (Arce \& Goodman 2002). For example, shocked outflow gas should be higher temperature than ambient gas, and the gas temperature should rise with the maximum outflow velocity (Lee et al. 2001). For optically thin gas, the excitation temperature may be related to the ratio of the line intensities by

$$
R_{21 / 10}=4 e^{-11 / T_{\mathrm{ex}}},
$$

where $R_{21 / 10}$ is the ratio of the ${ }^{12} \mathrm{CO}(2-1)$ to ${ }^{12} \mathrm{CO}(1-0)$ lines (Arce \& Goodman 2002). Observers typically assume that the high-velocity gas that constitutes the outflows is optically thin. We find that in many cases line ratios in the channels with $|v| \geqslant 2 \mathrm{~km} \mathrm{~s}^{-1}$ are above 1.0 , the line ratio limit in the optically thick case, indicating that the gas is at most marginally optically thick in the outflow.

Figure 13 shows that the estimated excitation temperatures are generally higher than the ambient gas temperature of $10 \mathrm{~K}$. The excitation temperatures also increase with time, which is expected if the shock strength and, hence, post-shock temperature increase with time. This effect also occurs in the simulations, although a rising gas temperature also results from

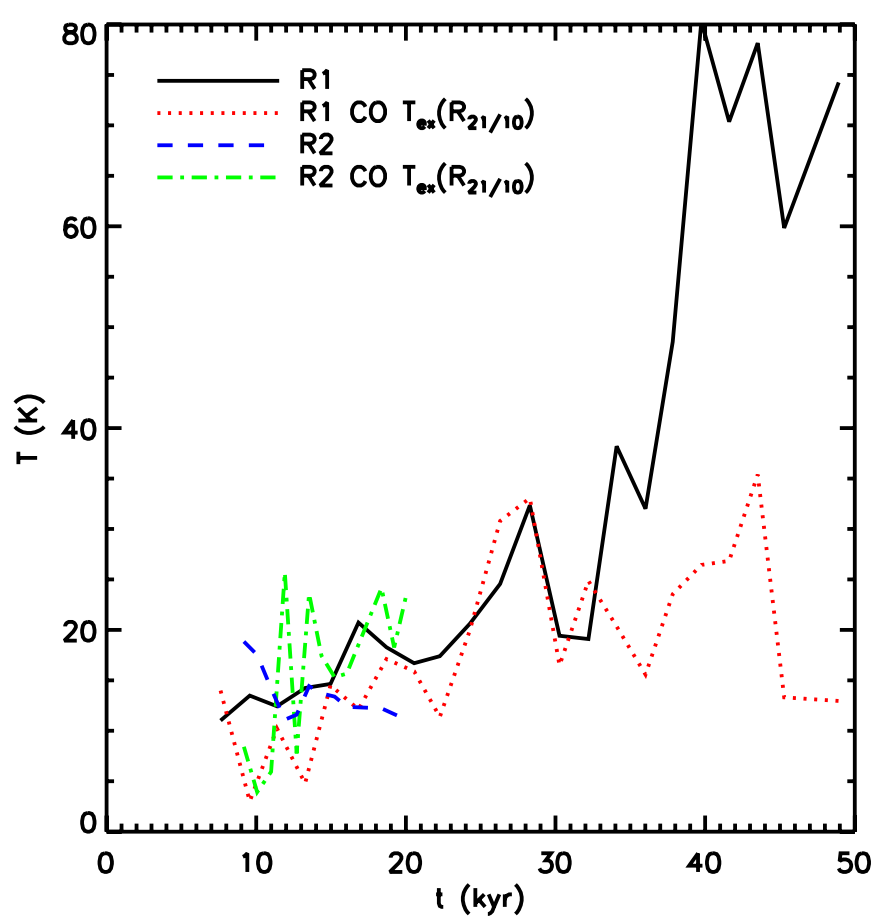

Figure 13. Outflow gas temperature vs. time when the outflow is inclined $45^{\circ}$ with respect to the line of sight. The $R 1$ and $R 2$ gas temperatures are computed as an average over the cells with velocities $\left|v_{45}\right| \geqslant 2 \mathrm{~km} \mathrm{~s}^{-1}$. The CO excitation temperature, $T_{\mathrm{ex}}$, is calculated using Equation (8) for an observation with $4^{\prime \prime}$ resolution.

(A color version of this figure is available in the online journal.)

increased protostellar heating (see OKMK09). The two causes are difficult to distinguish from a single average temperature value. However, $T_{\mathrm{ex}}$ does not appear to be well correlated with the actual gas temperatures in the simulation. The disagreement at later times is likely due to the increasing optical depth of the high-velocity gas. Hatchell et al. (1999) show that gas with $\tau=1$ and inferred $T_{\mathrm{ex}}=30 \mathrm{~K}$ under the assumption that the gas is optically thin will actually have $T_{\mathrm{ex}}=40 \mathrm{~K}$. The optically thin approximation increasingly underestimates temperatures for higher optical depths and gas temperatures.

\section{DISCUSSION}

Often only a single outflow lobe is observed (Arce et al. 2010; Ginsburg et al. 2011), creating uncertainty whether high gas velocities are instead due to coherent turbulent motion. We find that even with completely symmetric bipolar energy injection, interactions between the outflow and the turbulent envelope can result in significant asymmetry between the lobes. Combined with inclination effects, it is therefore quite possible for observations to identify only one component.

Seale \& Looney (2008), who include 12 outflows with only a single visible lobe in their photometrically identified sample, attribute asymmetry to inclination effects that increase obscuration of the far lobe by the core envelope. For outflows identified by high-velocity, coherent motion, the absence of a counterpart may be due to a combination of significant source advection, interference with the turbulent cloud velocities, or asymmetric dynamical or magnetic interaction with the envelope. Since dense, star-forming gas and young protostars are observed to move subsonically relative to their envelopes and the host cloud (Kirk et al. 2010; Offner et al. 2009a), obscuration and envelope interaction are the most likely causes of non-detected lobes. 
Outflow-envelope interactions are also largely responsible for the inferred outflow opening angle. Since the launching region and angle of the outflow remain fixed in our simulations, the significant angle broadening is a result of the outflow sweeping up successively larger solid angles of the core envelope. Over the course of the simulation, the mean density of the core does not decrease significantly. However, the outflow mass and momentum, which are coupled to the protostellar mass, increase with time. Once the outflow breaks out of the dense envelope, an increasing amount of gas on the edge of the cavity is swept up, broadening the cavity. Thus, current observational resolution is likely not yet probing the outflow launching region. Our hydrodynamic simulations are also in surprisingly good agreement with opening angles inferred from observations, suggesting that the details of the magnetic field evolution may play a sub-dominant role on these scales. Consequently, evolving opening angle trends cannot yet be used to distinguish between different theoretical models and instead reflect a generic characteristic of outflow-envelope interaction.

Exactly how outflows are responsible for driving turbulent motions in molecular clouds remains an open question in star formation. Some simulations of $\sim p c$ size clouds indicate that the momentum from outflows is sufficient to achieve quasisteady-state equipartition between turbulent and gravitational energy (Nakamura \& Li 2007; Wang et al. 2010). However, other simulations of jets indicate that well-collimated outflows drive supersonic turbulence very inefficiently, although they may contribute significant energy at lower velocities (Banerjee et al. 2007). However, observations of some molecular clouds suggest that the energy injected by outflows is insufficient by an order of magnitude or more than the observed cloud turbulence (Arce et al. 2010; Ginsburg et al. 2011, but see also Swift \& Welch 2008; Nakamura et al. 2011). Such estimations include corrections for inclination effects and mass underestimations that amount to a factor of four. Previous studies have found that neglecting low-velocity material may result in underpredicting the outflow mass by at least a factor of two (Margulis \& Lada 1985). For outflows identified with minimum velocities of $10 \mathrm{~km} \mathrm{~s}^{-1}$, the missing momentum may be even larger since outflows from the lowest mass sources and outflows aligned perpendicular to the line of sight may be missed altogether.

The method of AG01, which includes lower velocity gas, exhibits the best agreement with the launched mass. However, it is unclear how much of the low-velocity material, which dominates the mass estimate, is actually non-Gaussian turbulent core gas. The underestimation of outflow mass by both $\mathrm{CO}$ methods that employ a velocity cutoff underscores the large uncertainties underlying these derivations. Such techniques are likely more accurate at later stages when the core mass has declined and more gas has been swept up by the outflows. Nonetheless, this result has important implications for the derivation of outflow momentum. In a worst-case scenario, the masses of young, inclined outflows in optically thick regions are underestimated by factor of 50 .

Despite the severity of the problem, an order-of-magnitude discrepancy relative to corrections already applied by observers is unlikely. In addition, the momentum contributed by outflows from very young low-mass stars is likely small compared to outflows from higher mass sources. Consequently, the discrepancy we demonstrate here is unlikely to account for the turbulent deficit measured in clouds $\geqslant 10 \mathrm{pc}$. In addition, outflows must not only account for the magnitude of the energy injection but the appropriate range of scales (Matzner 2002; Swift \& Welch 2008). If measured outflow extents range from $\sim 0.1$ to $2 \mathrm{pc}$ then they will not contribute driving motions on larger scales. Magnetohydrodynamic simulations of outflow-driven turbulence confirm that the peak of the velocity power spectrum occurs at the maximum length scale of the driving outflows (Carroll et al. 2009, 2010).

\section{CONCLUSIONS}

We present results from self-gravitating, radiationhydrodynamic simulations of low-mass protostellar outflows using the AMR code ORION. We produce synthetic observations of the simulations in ${ }^{12} \mathrm{CO}$ and ${ }^{13} \mathrm{CO}$ in order to make direct comparisons with observations.

We formulate a quantitative prescription for measuring outflow opening angles and test that it reproduces previous estimates of observed outflow angles determined "by eye." Using this method, we find that both the simulation data and synthetic observations of the simulation data show opening angle evolution that is similar to trends found by Arce \& Sargent (2006). However, we show that beam resolution can significantly broaden the observed outflow angle and may broaden angles inferred by Arce \& Sargent (2006) by as much as a factor of two. Different inclinations with respect to the line of sight have a smaller effect on the measured angles.

We find that the interaction between the outflows and the turbulent core envelopes produces significant asymmetry and variation in outflow properties. Outflowing gas running into denser gas may be diverted or confined. This explains why observations may sometimes only identify a single outflow lobe. The clumpy outflow geometry in some observed outflows may even result from outflow-envelope interaction rather than variable mass accretion/ejection.

Using CO isotopologues we estimate the observed gas masses in two ways. First, we infer mass using $X_{\mathrm{CO}}$, the standard factor often used by extragalactic observers to convert between $\mathrm{CO}$ emission and molecular gas mass. Second, we apply the method of Arce \& Goodman (2001), who combine ${ }^{12} \mathrm{CO}(1-0)$ and ${ }^{13} \mathrm{CO}(1-0)$ data to obtain mass estimates. We find that although the mass of the high-velocity outflow component tracks the actual outflow mass, this gas is not a good estimate for the total integrated outflow mass. Even with a perfect conversion between $\mathrm{CO}$ emission and gas mass, outflow mass inferred only using emission from the high-velocity component underestimates the mass launched by a factor of $\sim 2-5$.

Masses derived from the $\mathrm{CO}$ emission, which is a poor tracer of gas mass in the optically thick limit, underestimate the actual outflow mass by an additional factor of 5-10. Ideally, as Arce \& Goodman (2001) suggest, lower velocity outflow gas can be included in the outflow estimate if the core mass can be modeled and subtracted from the emission. However, in the simulations the core mass distribution does not necessarily have a symmetric distribution of gas velocities, and so it is not well modeled with a simple functional form. Consequently, the more complicated method of Arce \& Goodman (2001) produces a larger but not necessarily more accurate outflow mass.

We find that the excitation temperatures derived from ${ }^{12} \mathrm{CO}$ are not necessarily well correlated with the simulation gas temperatures. However, they indicate that the high-velocity outflow gas is hotter than the ambient gas and that the gas temperature increases with time, which is consistent with the raw data from the simulations. 
In our simulations, the outflow launching region remains fixed throughout. Consequently, we conclude that observations of opening angle evolution do not probe the outflow launching mechanism and instead reflect the evolving interaction between the outflow and core envelope. Higher resolution observations in addition to simulations including the effects of magnetic fields will be needed to accurately investigate the physics of this innermost region.

We thank Eric Keto for assistance with MOLLIE and Andrew Cunningham for technical improvements to ORION. We also thank Tom Robitaille, Chris Beaumont, Eric Keto, Michelle Borkin, and Ned Ladd for helpful discussions and the referee Robi Banerjee for helpful comments. This research has been supported by the NSF through grants AST-0901055 (SSRO) and AST-0908159 (EJL). The simulations and data analysis were performed on the Odyssey supercomputing cluster at Harvard University.

\section{REFERENCES}

Arce, H. G., Borkin, M. A., Goodman, A. A., Pineda, J. E., \& Halle, M. W. 2010, ApJ, 715, 1170

Arce, H. G., \& Goodman, A. A. 2001, ApJ, 551, L171

Arce, H. G., \& Goodman, A. A. 2002, ApJ, 575, 928

Arce, H. G., \& Sargent, A. I. 2005, ApJ, 624, 232

Arce, H. G., \& Sargent, A. I. 2006, ApJ, 646, 1070

Arce, H. G., Shepherd, D., Gueth, F., et al. 2007, in Protostars and Planets V, ed. B. Reipurth, D. Jewitt, \& K. Keil (Tucson, AZ: Univ. Arizona Press), 245

Bally, J., Reipurth, B., Lada, C. J., \& Billawala, Y. 1999, AJ, 117, 410

Banerjee, R., Klessen, R. S., \& Fendt, C. 2007, ApJ, 668, 1028

Bontemps, S., Andre, P., Terebey, S., \& Cabrit, S. 1996, A\&A, 311, 858

Carolan, P. B., Khanzadyan, T., Redman, M. P., et al. 2009, MNRAS, 400, 78

Carolan, P. B., Redman, M. P., Keto, E., \& Rawlings, J. M. C. 2008, MNRAS, 383,705

Carroll, J. J., Frank, A., \& Blackman, E. G. 2010, ApJ, 722, 145

Carroll, J. J., Frank, A., Blackman, E. G., Cunningham, A. J., \& Quillen, A. C. 2009, ApJ, 695, 1376
Cernicharo, J., \& Reipurth, B. 1996, ApJ, 460, L57

Chernin, L. M., \& Masson, C. R. 1995, ApJ, 455, 182

Cunningham, A. J., Klein, R. I., Krumholz, M. R., \& McKee, C. F. 2011, ApJ, 740,107

Dame, T. M., Hartmann, D., \& Thaddeus, P. 2001, ApJ, 547, 792

Frerking, M. A., Langer, W. D., \& Wilson, R. W. 1982, ApJ, 262, 590

Ginsburg, A., Bally, J., \& Williams, J. 2011, MNRAS, in press (arXiv:1106.1433)

Glover, S. C. O., \& Mac Low, M. 2011, MNRAS, 412, 337

Hatchell, J., Fuller, G. A., \& Ladd, E. F. 1999, A\&A, 344, 687

Keto, E., \& Rybicki, G. 2010, ApJ, 716, 1315

Kirk, H., Pineda, J. E., Johnstone, D., \& Goodman, A. 2010, ApJ, 723, 457

Krumholz, M. R., McKee, C. F., \& Klein, R. I. 2004, ApJ, 611, 399

Ladd, E. F., Fuller, G. A., \& Deane, J. R. 1998, ApJ, 495, 871

Lee, C., Mundy, L. G., Reipurth, B., Ostriker, E. C., \& Stone, J. M. 2000, ApJ, 542,925

Lee, C., Stone, J. M., Ostriker, E. C., \& Mundy, L. G. 2001, ApJ, 557, 429

Li, Z., \& Shu, F. H. 1996, ApJ, 468, 261

Margulis, M., \& Lada, C. J. 1985, ApJ, 299, 925

Matzner, C. D. 2002, ApJ, 566, 302

Matzner, C. D., \& McKee, C. F. 1999, ApJ, 526, L109

Matzner, C. D., \& McKee, C. F. 2000, ApJ, 545, 364

Myers, P. C. 2009, ApJ, 706, 1341

Nakamura, F., Kamada, Y., Kamazaki, T., et al. 2011, ApJ, 726, 46

Nakamura, F., \& Li, Z. 2007, ApJ, 662, 395

Offner, S. S. R., Hansen, C. E., \& Krumholz, M. R. 2009a, ApJ, 704, L124

Offner, S. S. R., Klein, R. I., McKee, C. F., \& Krumholz, M. R. 2009b, ApJ, 703, 131

Pelletier, G., \& Pudritz, R. E. 1992, ApJ, 394, 117

Pineda, J. E., Caselli, P., \& Goodman, A. A. 2008, ApJ, 679, 481

Pollack, J. B., Hollenbach, D., Beckwith, S., et al. 1994, ApJ, 421, 615

Robitaille, T. P., Whitney, B. A., Indebetouw, R., Wood, K., \& Denzmore, P. 2006, ApJS, 167, 256

Rosen, A., \& Smith, M. D. 2004, MNRAS, 347, 1097

Seale, J. P., \& Looney, L. W. 2008, ApJ, 675, 427

Semenov, D., Henning, T., Helling, C., Ilgner, M., \& Sedlmayr, E. 2003, A\&A, 410,611

Shu, F. H., Najita, J., Ruden, S. P., \& Lizano, S. 1994, ApJ, 429, 797

Stone, J. M., Ostriker, E. C., \& Gammie, C. F. 1998, ApJ, 508, L99

Swift, J. J., \& Welch, W. J. 2008, ApJS, 174, 202

Wang, P., Li, Z., Abel, T., \& Nakamura, F. 2010, ApJ, 709, 27

Yu, K. C., Billawala, Y., \& Bally, J. 1999, AJ, 118, 2940 